Invited Editorial Focus

\title{
The Ultimate Murine Model of Immune Thrombocytopaenia
}

\author{
John W. Semple ${ }^{1}$ Rick Kapur ${ }^{2}$ \\ 1 Division of Hematology and Transfusion Medicine, Department of \\ Laboratory Medicine, Lund University, Lund, Sweden \\ 2 Department of Experimental Immunohematology, Sanquin \\ Research and Landsteiner Laboratory, Amsterdam UMC, University \\ of Amsterdam, Amsterdam, The Netherlands \\ Thromb Haemost 2019;119:353-354.
}

Immune thrombocytopaenia (ITP) is an autoimmune bleeding disorder characterized by platelet counts below $100 \times 10^{9} / \mathrm{L}$. Symptoms can manifest as petechiae, purpura, epistaxis, mucosal bleeding and, in rare cases, intracranial haemorrhage all of which lead to a significant reduction in the quality of life. Clinical approaches are complicated by the fact that patients with identical platelet counts can present with different clinical bleeding scenarios. In primary ITP, there is an isolated thrombocytopaenia, while in secondary ITP, the thrombocytopaenia is related to other autoimmune diseases (e.g. systemic lupus erythematosus) or other conditions such as chronic infections (e.g. Helicobacter pylori) ${ }^{1}{ }^{1}$ The pathophysiology of ITP is complex and heterogeneous 2,3 with an important role for anti-platelet autoantibodies ${ }^{4}$ and/ or T cell-mediated platelet destruction. ${ }^{5}$

To obtain insights into the complex nature of the pathophysiology, multiple murine models of ITP have been developed over the years. Generally, these include the passive ITP model, models of secondary ITP and a model of active ITP. The passive ITP model is based on injection of anti-platelet antibodies ${ }^{6}$ however, the thrombocytopaenia induced is not suitable to study chronic ITP and repeated anti-platelet injections are required to maintain a durable thrombocytopaenia. Moreover, the passive model is not appropriate for studying effector mechanisms related to $\mathrm{T}$ cell-mediated platelet destruction. Models of secondary ITP are complicated by the involvement of additional confounding autoimmune factors, and thus, are not ideal to study chronic ITP. In 2010, Chow et al ${ }^{7}$ introduced a murine model of active ITP that, for the first time, provided a tool which allowed in-depth investigations into the pathophysiology of chronic ITP. ${ }^{7}$ This model has greatly influenced the field as it accurately represents many of the immunopathogenic features of chronic ITP and has a strong resemblance to clinical features of human severe ITP. ${ }^{7-16}$ The model is based on the transfer of splenocytes from mice immune against platelet
Address for correspondence John W. Semple, PhD, Lund University, BMC C14, Klinikgatan 26, 221 84, Lund, Sweden

(e-mail: john_w.semple@med.lu.se; r.kapur@sanquin.nl).

CD61 into mice with severe combined immunodeficiency (SCID). Importantly, the thrombocytopaenia that developed in these mice was induced by effector mechanisms primarily mediated by both anti-platelet antibodies as well as on CD8 $+\mathrm{T}$ cells. Of interest, treatment of the SCID ITP mice with intravenous immunoglobulins (IVIgs) increased platelet counts and prevented bleeding mortality induced by antibody-mediated effector mechanisms but not by the $T$ cell-mediated indicating a differential response to IVIg therapy. ${ }^{7}$

In this issue of Thrombosis and Haemostasis, Li et al further illustrate the importance of the active ITP model by changing the antigenic system and immunizing CD41-knockout (KO) mice with platelets of wild-type mice and subsequently transferring these splenocytes into SCID mice. ${ }^{17}$ An advantage of using CD41-KO mice instead of the CD61-KO mice is that CD41 is a megakaryocyte- and platelet-specific marker, whereas CD61 is not only expressed by platelets but also present on other cells including endothelial cells. Additionally, placental defects have been described in CD61-KO females with an occurrence of post-natal haemorrhage. ${ }^{18}$ Indeed, Li et al demonstrated that loss of CD41 did not affect the survival of embryos or pups. More importantly, they have convincingly validated this new model by assessing platelet counts, antibody levels in serum and bone marrow megakaryocyte numbers in the SCID ITP mice. The SCID mice that received splenocytes from immunized CD41-KO mice suffered from thrombocytopaenia that persisted until day 21 and from haemorrhage in the intestines, lungs, brain and abdomen. Together with the thrombocytopaenia, increased serum anti-platelet antibodies were also detected which were likely responsible for the platelet destruction. Furthermore, reduced numbers of mature megakaryocytes were observed in the bone marrow of the SCID ITP mice which likely reduced platelet production as seen in human ITP. In addition, when the immune splenocytes were first depleted of CD19+ B cells received

January 22, 2019

accepted

January 22, 2019 (c) 2019 Georg Thieme Verlag KG Stuttgart · New York
DOI https://doi.org/ 10.1055/s-0039-1678697. ISSN 0340-6245. 
before transfer, the SCID mice developed severe thrombocytopaenia indicating the occurrence of $\mathrm{T}$ cell-mediated platelet destruction. Alternatively, when the transferred splenocytes were first depleted of CD8 $+\mathrm{T}$ cells, the number of mature megakaryocytes in the bone marrow significantly increased, indicating that $\mathrm{CD} 8+\mathrm{T}$ cells may be impairing bone marrow megakaryocyte maturation. Since antibody-mediated platelet destruction in ITP is presumed to occur through phagocytosis in the spleen, the authors then examined the contribution of the spleen in the model. Compared with non-splenectomized SCID mice, transfer of immunized splenocytes into splenectomized mice showed a significant recovery of platelet counts by day 21 in combination with a reduction of anti-CD41 antibody levels. This suggests that the spleen is the major site of both anti-platelet antibody production and platelet destruction in murine ITP.

In summary, Li et al confirm the importance of the murine model of active ITP ${ }^{7}$ by using a different platelet antigenic system. ${ }^{17}$ This new model is platelet-specific and demonstrates both antibody- as well as $\mathrm{T}$ cell-mediated platelet destruction making it a new valuable tool for in-depth investigations into the pathophysiology of chronic ITP.

Conflict of Interest

None declared.

\section{References}

1 Rodeghiero F, Stasi R, Gernsheimer T, et al. Standardization of terminology, definitions and outcome criteria in immune thrombocytopenic purpura of adults and children: report from an international working group. Blood 2009;113(11):2386-2393

2 Zufferey A, Kapur R, Semple JW. Pathogenesis and therapeutic mechanisms in immune thrombocytopenia (ITP). J Clin Med 2017;6(02):E16

3 McKenzie CG, Guo L, Freedman J, Semple JW. Cellular immune dysfunction in immune thrombocytopenia (ITP). Br J Haematol 2013;163(01):10-23

4 Harrington WJ, Minnich V, Hollingsworth JW, Moore CV. Demonstration of a thrombocytopenic factor in the blood of patients with thrombocytopenic purpura. J Lab Clin Med 1951;38(01):1-10

5 Olsson B, Andersson PO, Jernås M, et al. T-cell-mediated cytotoxicity toward platelets in chronic idiopathic thrombocytopenic purpura. Nat Med 2003;9(09):1123-1124
6 Crow AR, Song S, Semple JW, Freedman J, Lazarus AH. IVIg inhibits reticuloendothelial system function and ameliorates murine passive-immune thrombocytopenia independent of anti-idiotype reactivity. Br J Haematol 2001;115(03):679-686

7 Chow L, Aslam R, Speck ER, et al. A murine model of severe immune thrombocytopenia is induced by antibody- and CD8+ T cell-mediated responses that are differentially sensitive to therapy. Blood 2010;115(06):1247-1253

8 Aslam R, Hu Y, Gebremeskel S, et al. Thymic retention of CD4+CD25+FoxP3+ T regulatory cells is associated with their peripheral deficiency and thrombocytopenia in a murine model of immune thrombocytopenia. Blood 2012;120(10): 2127-2132

9 Aslam R, Kapur R, Segel GB, et al. The spleen dictates platelet destruction, anti-platelet antibody production, and lymphocyte distribution patterns in a murine model of immune thrombocytopenia. Exp Hematol 2016;44(10):924-930

10 Guo L, Kapur R, Aslam R, et al. Antiplatelet antibody-induced thrombocytopenia does not correlate with megakaryocyte abnormalities in murine immune thrombocytopenia. Scand J Immunol 2018;88(01):e12678

11 Guo L, Kapur R, Aslam R, et al. CD20+ B-cell depletion therapy suppresses murine CD8+ T-cell-mediated immune thrombocytopenia. Blood 2016;127(06):735-738

12 Guo L, Yang L, Speck ER, et al. Allogeneic platelet transfusions prevent murine T-cell-mediated immune thrombocytopenia. Blood 2014;123(03):422-427

13 Kapur R, Catalina MD, Aslam R, Speck ER, Francovitch RF, Semple JW. A highly purified form of staphylococcal protein A alleviates murine immune thrombocytopenia (ITP). Br J Haematol 2018;183 (03):501-503

14 Kapur R, Aslam R, Kim M, et al. Thymic-derived tolerizing dendritic cells are upregulated in the spleen upon treatment with intravenous immunoglobulin in a murine model of immune thrombocytopenia. Platelets 2017;28(05):521-524

15 Hou Y, Feng Q Xu M, et al. High-dose dexamethasone corrects impaired myeloid-derived suppressor cell function via Ets1 in immune thrombocytopenia. Blood 2016;127(12):1587-1597

16 Zufferey A, Speck ER, Machlus KR, et al. Mature murine megakaryocytes present antigen-MHC class I molecules to T cells and transfer them to platelets. Blood Adv 2017;1(20):1773-1785

17 Li X, Wang SW, Feng Q, et al. Novel murine model of immune thrombocytopaenia through immunized CD41 knockout mice. Thromb Haemost 2019;119(03). Doi: 10.1055/s-0038-1677032

18 Hodivala-Dilke KM, McHugh KP, Tsakiris DA, et al. Beta3-integrindeficient mice are a model for Glanzmann thrombasthenia showing placental defects and reduced survival. J Clin Invest 1999;103 (02):229-238 